Herausgegeben von Martin Doll und Oliver Kohns

\title{
DIE IMAGINÄRE DIMENSION DER POLITIK
}

\author{
WILHELM FINK
}


Gefördert vom

Fonds National de la

Recherche Luxembourg

Die Verantwortung für den Inhalt der Veröffentlichung liegt bei den Autoren.

Bibliografische Informationen der Deutschen Nationalbibliothek: Die Deutsche Nationalbibliothek verzeichnet diese Publikation in der Deutschen Nationalbibliografie; detaillierte Daten sind im Internet über http://dnb.d-nb.de abrufbar.

Alle Rechte, auch die des auszugweisen Nachdrucks, der fotomechanischen Wiedergabe und der Übersetzung vorbehalten. Dies betrifft auch die Vervielfältigung und Übertragung einzelner Textabschnitte, Zeichnungen oder Bilder durch alle Verfahren wie Speicherung und Übertragung auf Papier, Transparente, Filme, Bänder, Platten und andere Medien, soweit es nicht $\$ 53$ und 54 UrhG ausdrücklich gestatten.

(C) 2014 Wilhelm Fink Verlag, München

Wilhelm Fink GmbH \& Co. Verlags-KG, Jühenplatz 1,

D-33098 Paderborn, Internet: www.fink.de

Lektorat: Martin Doll, Oliver Kohns \& Christoph Roolf

Gestaltung und Satz: Sichtvermerk

Printed in Germany, Herstellung: Ferdinand Schöningh

GmbH \& Co. KG, Paderborn

ISBN 978-3-7705-5487-4 


\section{INHALT}

Martin Doll \& Oliver Kohns

7 Außer-sich-Sein: Die imaginäre Dimension der Politik. Einleitung

Oliver Kohns

19 Die Politik des "politischen Imaginären«

Martin Doll

49 Theorie und Genealogie des Techno-Imaginären: Social Media zwischen >Digital Nation politischem Pluralismus

Leander Scholz

91 Die Erhabenheit des Feldherrn. Imagination und politische Ordnung bei Castoriadis und Kant

Harun Maye

113 Von der Imagination zum Imaginären?

Ein Kommentar zur Begriffsgeschichte und Theorie des Imaginären bei Wolfgang Iser und Karl Ludwig Pfeiffer

Martin Roussel

135 Äquivokation. Die Sprache und das Imaginäre Randbemerkungen zu Ricœur und Derrida

Rolf Parr

165 Diskursive Positionen als Prismen des politisch Imaginären?

Jan Lohl

181 Die Nation als imaginäre Gemeinschaft. Ein psychoanalytischer Beitrag zur Nationalismusforschung am Beispiel der Konstitution nationaler Identität nach der deutschen Einheit 
Romain Jobez

215 Das Theater des Imaginären:

Lefort liest Gramsci liest Machiavelli

Benjamin Wihstutz

229 Der Streit um die Bühne:

Theatralität im politischen Denken Jacques Rancières

Jan Völker

257 Politik ohne Staat.

Zu Badious Rekonstruktion des Imaginären

290 Verzeichnis der Autoren 
Martin Doll und Oliver Kohns

\section{AUSSER-SICH-SEIN: \\ DIE IMAGINÄRE DIMENSION DER POLITIK EINLEITUNG}

Seit einigen Jahren wird dem (politik)wissenschaftlichen Blick auf politische Strukturen und Organisationsformen eine kulturwissenschaftliche Betrachtung der Politik entgegengesetzt, die anstelle der Analyse der vielfältigen Formen der Organisation von Politik nach Momenten und Möglichkeiten der Gründung von politischen Gemeinwesen fragt. Eine bedeutende Rolle spielt hierbei ein Begriff, der mittelbar den Vermögenslehren des 18. Jahrhunderts entlehnt ist: der Begriff des Imaginären bzw. der Imagination, der Einbildungskraft. Aus verschiedenen theoretischen Perspektiven beschreiben kulturwissenschaftliche Ansätze so ein politisches Imaginäres. Die Annahme etwa der Forschergruppe um Albrecht Koschorke lautet, dass »sich das Gemeinwesen in Bildern der Einheit vergegenwärtigt, die seinen Mitgliedern eine substanzielle Teilhabe am Sozialen verheißen «, und dass nur unter diesen Umständen das Gemeinwesen mehr sein könne »als eine Summe von Individuen, die unter kontingenten Umständen zusammengebracht worden sind. « ${ }^{1}$ Behauptet wird damit die Notwendigkeit einer politischen »Funktion der imaginären Ganzheitsstiftung «. ${ }^{2}$ Diese Setzung impliziert weitreichende Vorannahmen. Betrachtet man Politik als Raum eines >sachlichen $<$, d.h. wesentlich rational bestimmten Denkens und Handelns, dann erscheint das Imaginäre bzw. die Imagination lediglich als kontrafaktischer Bereich des bloß Möglichen, als tendenziell unpolitisch oder vorpolitisch: bestenfalls als utopische Ausbuchstabierung einer zukünftigen Realpolitik,

1 Thomas Frank, Albrecht Koschorke, Susanne Lüdemann, Ethel Matala de Mazza, Des Kaisers neue Kleider. Über das Imaginäre politischer Herrschaft. Texte, Bilder, Lektüren, Frankfurt a. M. 2002, S.78.

2 Ebd. 
schlimmstenfalls als ein Supplement des Politischen z. B. in Form von >Symbolpolitik $<$, die dem >echten $<$ politischen Handeln seine reine >Inszenierung< entgegensetzt. ${ }^{3}$ Nimmt man jedoch im Sinne der kulturwissenschaftlichen Theorie an, dass politische Institutionen und politisches Handeln erst durch eine »imaginäre Ganzheitsstiftung « ermöglicht werden, dann wird das Imaginäre nicht als bloßer >Schein< konzipiert, sondern es begründet gesellschaftlichpolitische Institutionen und damit jede >reale< Politik (und steht so jenseits der Differenz >wahr / > unwahr $)$. In diesem Sinn schreibt die Gruppe um Koschorke: »Neuere Theorien haben [...] zu der Einsicht geführt, dass die soziale und politische Ordnung selbst auf einer Ordnung des Imaginären beruht, welche Dichotomien vom Typ Basis/Überbau oder Realität/Fiktion durchkreuzt. « ${ }^{4}$

An diese Forschungsperspektive schließt der vorliegende Band an. Die meisten in diesem Zusammenhang erschienenen Arbeiten betonen zu Recht die historische Dimension des Themas und binden die Analyse des politischen Imaginären etwa an die »Geschichte des Staates $\aleph^{5}$ und seiner imaginären Repräsentationen. Neben dieser historischen Dimension kann auch nach der theoretischen Ausgestaltung des Konzepts des politischen Imaginären gefragt werden. Dabei lassen sich nicht nur Formen des politischen Imaginären jenseits seiner Funktionalisierung für den Staat oder die Figur des (monarchischen) Souveräns analysieren, sondern das politische Imaginäre kann auch grundsätzlich anders gefasst werden, um stärker demokratische Strukturen (auch mit Blick auf mediale Dispositive) in den Blick zu bekommen. Zugleich kann und muss die Theorie des politischen Imaginären selbst in ihrer historischen Dimension analysiert werden: Das Imaginäre hat seine eigene Genealogie, und jede Theorie des politischen Imaginären schreibt sich nolens volens in weitverzweigte Traditionslinien ein, die jeweils zahlreiche Axiome und Voraussetzungen mit sich bringen. Der Band Die imaginäre Dimension der Politik fragt daher zugleich nach der Theorie des politischen Imaginären und nach ihrer (Vor-)Geschichte.

3 Vgl. beispielsweise Oliver Lepsius, »Die Causa Guttenberg als interdisziplinäre Fallstudie - eine Einleitung «, in: ders., Reinhart Meyer-Kalkus (Hg.), Inszenierung als Beruf. Der Fall Guttenberg, Berlin 2011, S. 7-17, hier: S. $13 \mathrm{f}$.

4 Albrecht Koschorke, Susanne Lüdemann, Thomas Frank, Ethel Matala de Mazza, Der fiktive Staat. Konstruktionen des politischen Körpers in der Geschichte Europas, Frankfurt a. M. 2007, S.11.

5 Ebd. 
Eine Genealogie des politischen Imaginären müsste mindestens bis zu Rousseaus Philosophie ausgreifen. Diese enthält eine Theorie der imagination, der Einbildungskraft. Sie wird im 18. Jahrhundert im Rahmen der Vermögenslehre interpretiert: Als Vermögen gilt hier jede dem menschlichen Geist zuschreibbare Kraft, Wirkungen hervorzubringen, sowohl nach außen als auch in Bezug auf sich selbst. ${ }^{6}$ Die Einbildungskraft wird im 18. Jahrhundert (noch bei Kant) definiert als »Vermögen der Anschauungen auch ohne Gegenwart des Gegenstandes «.? Durch die Einbildungskraft stellt sich das Subjekt quasi-sinnliche Wahrnehmungen in Abwesenheit des Wahrgenommenen vor - oder wird von solchen Wahrnehmungen potenziell ohne Absicht betroffen. Die Einbildungskraft ist so zwar ein Teil der Vermögenslehre, schert jedoch aus allen Kategorien aus: Sie gehört zur sinnlichen Wahrnehmung und zum Verstand, sie ist immer zugleich aktiv und passiv und also gleichzeitig Vermögen und Unvermögen, vom Subjekt gesteuert und das Subjekt steuernd. ${ }^{8}$

Trotz dieser Ambivalenz erhält das Vermögen bei Rousseau eine zentrale Bedeutung, insofern er ihm das Potenzial zuschreibt, den Menschen für das Zusammenleben mit anderen Menschen zu öffnen. Die imagination erscheint als eine Kraft, die Gesellschaftlichkeit und Politik möglich macht. »Die gesellschaftlichen Zuneigungen [affections sociales] entfalten sich in uns nur mit unserem Erkenntnisvermögen [lumières] «, schreibt Rousseau: »Derjenige, der sich nichts vorstellt, fühlt nur sich selbst; er ist allein inmitten des Menschengeschlechts [Celui qui n'imagine rien ne sent que luimême; il est seul au milieu du genre humain]. ${ }^{9}$

$6 \gg$ Facultas, das Vermögen, wird von denen Medicis vor die sonderbare Würckung der Seelen genommen, welche sie in diesem oder jenem Gliede, oder auch in sich selbst exerciret." (Johann Heinrich Zedler, Grosses vollständiges Universal-Lexikon aller Wissenschafften und Künste [1732-1750], Bd.1-64, Graz 1961, Bd.9, Sp.69)

7 Immanuel Kant, »Anthropologie in pragmatischer Hinsicht «, in: ders., Werke in sechs Bänden, hg. von Wilhelm Weischedel, Darmstadt 1983, Bd.6, S.399-690, hier: S. 466.

8 Vgl. Wolfgang Iser, »Fiktion/Imagination «, in: Ulfert Ricklefs (Hg.), Fischer Lexikon Literatur, Bd.1-3, Frankfurt a. M. 1996, Bd.1, S. 662-679, hier: S.670, und Lorraine Daston, »Angst und Abscheu vor der Einbildungskraft in der Wissenschaft «, in: dies., Wunder, Beweise und Tatsachen. Zur Geschichte der Rationalität, Frankfurt a. M. 2001, S. 99-125, hier: S. $106 \mathrm{f}$.

9 Jean-Jacques Rousseau, »Versuch über den Ursprung der Sprachen, in dem von der Melodie und der musikalischen Nachahmung 
Der besondere Stellenwert der imagination in Rousseaus politischer Theorie erklärt sich durch ihre Funktion als Bedingung der Möglichkeit des Mitleidens mit anderen. Im zweiten Diskurs bezeichnet Rousseau das Mitleid als die »einzige natürliche Tugend [la seul vertu Naturelle] «, ${ }^{10}$ insofern bereits der Naturmensch dazu befähigt sei. Obwohl selbst nicht moralisch motiviert, konstituiert es moralisches Handeln: Es »mäßigt « den »Eifer für das eigene Wohlsein «, ${ }^{11}$ die natürliche Selbstliebe, und macht den Menschen aufgeschlossen für soziale Beziehungen zu anderen Menschen. Die Fähigkeit zum Mitleid wird so zur Grundlage jeder Gesellschaftlichkeit: »Die Gewogenheit und die Freundschaft selbst entspringen [...] aus einem beständigen Mitleiden [une pitié constante] «.12 Dabei betont Rousseau, dass das Mitleid nur durch die Einbildungskraft initiiert wird: »Das Mitleid [pitié], obgleich dem menschlichen Herzen von Natur eingegeben, bliebe ohne Zutun der Einbildungskraft, die es auslöst [qui la met en jeu], für immer untätig [inactive]. « ${ }^{13} \mathrm{Als}$ Vermögen, sich das Leiden anderer vorzustellen, sich mit leidenden Menschen zu identifizieren, ermöglicht das durch die imagination angeregte Mitleid eine grundlegende Selbst-Alteration des Menschen. Die imagination vermag es, jeden einzelnen Menschen »aus sich selbst hinauszuversetzen [transporter hors de lui] «. ${ }^{14}$ Der Mensch ist für und seit Rousseau grundsätzlich außer sich. ${ }^{15}$

die Rede ist «, in: ders., Sozialphilosophische und Politische Schriften, in Erstübersetzung von Eckhart Koch, Dietrich Leube, Melanie Walz und Hans Zischler sowie bearbeiteten und ergänzten Übersetzungen aus dem 18. und 19. Jahrhundert, Düsseldorf 2001, S.165-221, hier: S. 186 (Übersetzung modifiziert).

10 Jean-Jacques Rousseau, »Abhandlung über den Ursprung und die Grundlagen der Ungleichheit unter den Menschen«, in: ders., Schriften, hg. von Henning Ritter, Frankfurt a. M. 1988, Bd.1, S.165302, hier: S. 218.

11 Ebd.

12 Ebd., S. 220.

13 Rousseau, »Versuch über den Ursprung der Sprachen« (wie Anm. 9), S. 186.

14 Jean-Jacques Rousseau, Emile oder Von der Erziehung. Emile und Sophie oder Die Einsamen. Emile oder Von der Erziehung in der deutschen Erstübertragung von 1762, nach der Edition Duchesne überarbeitet von Siegfried Schmitz, Düsseldorf/Zürich 1997, S. 273.

15 Vgl. Oliver Kohns, Die Verrücktheit des Sinns. Wahnsinn und Zeichen bei Kant, E. T.A.Hoffmann und Thomas Carlyle, Bielefeld 2007, S. 44 f. sowie Roberto Esposito, Communitas. Ursprung und Wege der 
Diese Alterierung des Selbst lässt sich, wenn Rousseau davon spricht, dass die gesellschaftlichen Zuneigungen »sich in uns nur mit unserem Erkenntnisvermögen « entfalten, als »Produkt einer allmählichen Entwicklung ${ }^{16}{ }^{16}$ entweder in eine individuelle Psychogenese (in Emile) oder als Erweiterung des Vorstellungsraums in die Genese der Gattung des Menschen einbetten. Das Mitleid, schreibt Rousseau, ist »ein Gefühl, das bei einem Wilden [l'homme sauvage] dunkel und lebhaft [obscur et vif], bei dem gesitteten Menschen [l'homme civil] hingegen aufgeklärt, aber schwach [développé, mais faible] sein muß «. ${ }^{17}$ Die menschheitsgeschichtliche Entwicklungslinie führt also vom »Wilden« zum »zivilisierten« Menschen, zeichnet sich aber nicht durch eine lineare Fortschrittslogik aus. Die Wirkung der Einbildungskraft dehnt sich zwar auf eine größere Anzahl von Menschen aus: Der »Wilde « lebt Rousseau zufolge ohne sozialen Kontakt zu anderen Menschen, ${ }^{18}$ der $»$ zivilisierte « Mensch entwickelt dagegen die Vorstellung einer allgemeinen »Humanität ", die Kraft des Mitleids verringert sich in diesem Prozess jedoch. ${ }^{19}$ Notwendig für eine wirkliche gemeinschaftliche Sozialität wäre in Rousseaus Perspektive daher eine wie auch immer geartete Beschränkung des Umfangs des Mitleids: »Man muß in gewisser Weise die Anteilnahme und das Mitleiden begrenzen und verdichten, um ihm Wirksamkeit zu verleihen. ${ }^{20}$

Ausgehend von Rousseau wird die Einbildungskraft immer wieder zum zentralen Vermögen der Vergesellschaftung gemacht, werden Gesellschaftlichkeit und politischer Zusammenhalt vom Potenzial der Einbildungskraft abgeleitet, die Menschen »aus sich hinauszuversetzen«. In der politischen Theorie des 20. Jahrhunderts wird die Idee der Fundierung der Soziabilität durch das Vermögen der Einbildungskraft dann neu ausformuliert. Der Rahmen

Gemeinschaft, übers. von Sabine Schulz und Francesca Raimondi, Berlin 2004, S. $93 \mathrm{f}$.

16 Iring Fetscher, Rousseaus politische Philosophie. Zur Geschichte des demokratischen Freiheitsbegriffs, 3. Aufl., Frankfurt a. M. 1993, S. 41.

17 Rousseau, »Abhandlung über den Ursprung und die Grundlagen der Ungleichheit unter den Menschen« (wie Anm.10), S. 220.

18 Vgl. ebd., S.222: "Sie hatten nicht die mindeste Gemeinschaft unter sich [ils n'avoient entre eux aucune espéce de commerce] «.

19 Vgl. Jean-Jacques Rousseau, »Abhandlung über die politische Ökonomie«, in: ders., Sozialphilosophische und Politische Schriften (wie Anm.9), S. 227-265, hier: S. 241.

20 Ebd. 
der Vermögenslehre des 18. Jahrhundert wird dabei gesprengt. Spätestens seitdem Nietzsche die Vermögenslehre Kants als eine »lustige niaiserie allemande ${ }^{21}{ }^{21}$ verspottete, hat das Konzept des Vermögens seine Autorität als Ordnungsschema des menschlichen Geistes eingebüßt. Die Einbildungskraft schreibt sich im 20. Jahrhundert jedoch als das Imaginäre weiter. Lacans Modell der Psyche greift die Bestimmung der Einbildungskraft bei Schelling als »Kraft der Ineinsbildung «, ${ }^{22}$ also der Synthesen auf, insofern das Imaginäre hier eine »Ordnung der Spiegelbilder, der Identifizierungen und der wechselseitigen Abhängigkeiten ${ }^{23}{ }^{23}$ innerhalb des Ich darstellt: Bei der primären Identifikation während des Spiegelstadiums wird das Subjekt von seinem eigenen Bild gebannt und erfährt sein IdealIch als »Versprechen zukünftiger Ganzheit «. ${ }^{24}$ Im Unterschied zu Schelling ist das Imaginäre dabei kein Vermögen mehr, über das ein Subjekt gebieten könnte, sondern vielmehr ein Element der Struktur, die das Subjekt konstituiert. Es geht also nicht mehr um die Vorgängigkeit eines Bilder oder Vorstellungen erzeugenden Subjekts, sondern umgekehrt darum, das Ich als Effekt einer Identifizierung mit etwas, das ihm äußerlich ist, zu beschreiben.

Bei Castoriadis wird das Imaginäre wieder ein Vermögen, und ein ausdrücklich politisches Vermögen, insofern es hier die Struktur der Gesellschaft und seiner politischen Institutionen (z. B. der des Staates) bestimmt. Dabei unterscheidet Castoriadis eine >radikale< von einer >aktualen< Einbildungskraft: ${ }^{25}$ Während erstere gesellschaftliche Strukturen überhaupt erst vorstellbar und damit möglich macht, ist letztere auf den Fundus von Vorstellungen einer bereits institutionalisierten Ordnung beschränkt. Der Vorstellungskraft in ihrer radikalen Dimension kommt daher auch

21 Vgl. Friedrich Nietzsche, »Jenseits von Gut und Böse«, in: ders., Sämtliche Werke. Kritische Studienausgabe in 15 Einzelbänden, hg. von Giorgio Colli und Mazzino Montinari, Berlin / New York / München 1988, Bd.5, S. 9-243, hier: S. 24 .

22 Friedrich Wilhelm Joseph Schelling, »Philosophie der Kunst« (1802/1803), in: ders., Ausgewählte Schriften in 6 Bänden, Frankfurt a. M. 1985, Bd.2, S.181-565, hier: S. 214.

23 Malcolm Bowie, Lacan, übers. von Klaus Laermann, Göttingen 1997, S. 90.

24 Dylan Evans, Wörterbuch der Lacanschen Psychoanalyse, Wien 2002, S. 279 .

25 Vgl. Cornelius Castoriadis, Gesellschaft als imaginäre Institution. Entwurf einer politischen Philosophie, übers. von Horst Brühmann, Frankfurt a.M. 1990, S. 218. 
das Potenzial zu, die Kontingenz jeder Form von erstarrter Institutionalisierung denkbar zu machen und zu ihrer Verflüssigung beizutragen. In diesem Fall gerät nicht nur der einzelne Mensch, wie bei Rousseau, qua imagination außer sich, sondern die Einrichtung des Gesellschaftlichen selbst wird dynamisch und transzendierbar.

Dezidiert von einer grundsätzlich imaginären Verfasstheit jeder Form von politischer Gemeinschaft geht auch Benedict Andersons These der »imagined communities « aus: »In der Tat sind alle Gemeinschaften, die größer sind als die dörflichen mit ihren Face-to-face-Kontakten, vorgestellte Gemeinschaften [imagined communities]. ${ }^{26}$ Das Buch (und sein zentrales Axiom) war außerordentlich einflussreich und gilt inzwischen als kanonischer >Klassiker< der Kulturwissenschaften. ${ }^{27}$ So erfolgreich konnte Anderson möglicherweise nur sein, weil er in zentralen Punkten verblüffend traditionell bleibt: Der Begriff der »imagined communities « schließt an Rousseaus These der Vergemeinschaftung durch die Einbildungskraft an, insofern doch wieder davon ausgegangen wird, dass einzelne Subjekte jeweils eigene Vorstellungen, die aber einander gleichen, hervorbringen. Die kulturwissenschaftliche Forschung der Gegenwart operiert so mit Theorien der Imagination und des Imaginären, die aus dem 18. Jahrhundert stammen. Eine neue Perspektive gewinnt Anderson der Frage des politischen Imaginären allerdings ab, indem er die vorgestellten Gemeinschaften - in der Tradition materialistischen Denkens - aus der gesellschaftlichen Entwicklung von Medientechnologien ableitet, insbesondere vermittelt durch das Druckwesen im 18. Jahrhundert. Vor allem Roman und Zeitung lieferten als Massenmedien Anderson zufolge, da sie die Vorstellung von Gleichsprachigkeit und Gleichzeitigkeit ermöglichten, den Grund für die »Geburt der vorgestellten Gemeinschaft der Nation «: ${ }^{28}$ der Roman durch sein Darstellungsverfahren von Simultaneität (»Wie Gott beobachten

26 Benedict Anderson, Die Erfindung der Nation. Zur Karriere eines folgenreichen Konzepts, erweiterte Ausgabe, übers. von Benedikt Burkard und Christoph Münz, Berlin 1998, S.15.

27 Vgl. Jonathan Culler, »Anderson and the Novel«, in: ders., Pheng Cheah (Hg.), Grounds of Comparision. Around the Work of Benedict Anderson, New York/London 2003, S. 29-52, hier: S.29: »Benedict Anderson's Imagined Communities: Reflections on the Origins and Spread of Nationalism has, in the past decade, become a classic of the humanities and social sciences."

28 Anderson, Die Erfindung der Nation (wie Anm.26), S. 29. 
die Leser gleichzeitig, wie A mit C telefoniert, B einkauft und D Billard spielt $«{ }^{29}$ ), die Zeitung durch die Fiktion ihres »praktisch gleichzeitige [n] Konsum[s] «.30 Vor allem letztere Erfahrung führt dazu, dass sich die einzelnen Zeitungsleser während ihrer konkreten Lektürepraxis wie in einem filmischen Rückzoom über sich hinausgehend als Teil einer größeren sozialen Einheit mit anderen Mitgliedern imaginieren konnten.

Die imaginären Gemeinschaften erscheinen in Andersons Theorie folglich als unbewusst strukturiert, so dass etwa »Millionen von Menschen für so begrenzte Vorstellungen weniger getötet haben als vielmehr bereitwillig gestorben sind. ${ }^{31}$ Dennoch rückt Anderson dies ausdrücklich nicht in den Kontext >falscher< Phantasmen, um diesen dann eine >echte< Erfahrung gegenüberzustellen. ${ }^{32}$ Vielmehr lassen sich die von ihm beschriebenen $»$ Wahrnehmungsformen « an aktuelle kulturwissenschaftliche Diskurse anschließen, in denen zum einen die affektive Dimension des politischen Imaginären hervorgehoben und zum anderen davon ausgegangen wird, dass auch »Geschichten Modelle und Denkfiguren bereitstellen, die schöpferisch auf den politischen Prozess zurückwirken . $^{33}$

Der vorliegende Band versucht nicht - ebenso wenig wie dieser Text-, eine vollständige >Geschichte $<$ des Wechselverhältnisses zwischen Einbildungskraft, der imagination bzw. dem Imaginären und der Genese politischer Strukturen zu schreiben. Es wird nicht angestrebt, wie es immer so schön heißt, eine bestehende Forschungslücke restlos zu schließen, d.h. eine handbuchartige Synthese dessen zu bieten, was man >heute< über die Imagination und das Imaginäre wissen sollte, oder sogar ein letztgültiges

29 Ebd., S.30.

30 Ebd., S.37.

31 Ebd., S.16.

32 Vgl. ebd., S. 15 .

33 Koschorke u.a., Der fiktive Staat (wie Anm.4), S.57, vgl. auch S. 62-64, Anderson analogisiert die durch den Zeitungsleser vorgestellte gemeinschaftliche Verbundenheit folgendermaßen mit dem Roman: »Wie bei Noli Me Tangere sickert die Fiktion leise und stetig in die Wirklichkeit ein und erzeugt dabei jenes bemerkenswerte Vertrauen in die anonyme Gemeinschaft, welche das untrügliche Kennzeichen moderner Nationen ist « (Anderson, Die Erfindung der Nation [wie Anm.26], S.37). 
Konzept des politischen Imaginären selbst. Die Autoren erhellen daher schlaglichtartig - und durchaus auch kritisch - bestimmte Blickpunkte einzelner Theoretiker und Theorien. In diesem $\mathrm{Zu}-$ sammenhang stellen sie auch die Frage, ob und wie die genannten Kategorien oder vielmehr die Betrachtung der »imaginären Dimension der Politik « für die weitere Forschung überhaupt lohnend sein können. Da es keine politische Theorie geben kann, die nicht auch ihrerseits politisch ist, also eine politische Position einnimmt, fragen zahlreiche Beiträge schließlich auch nach der politischen Positionierung der Theorie(n) über das politische Imaginäre.

Oliver Kohns eröffnet den Band mit einer kritischen Perspektive auf die (deutschsprachige) kulturwissenschaftliche Forschung zum politischen Imaginären. Jede Konzeption des politischen Imaginären ist stets ihrerseits politisch, lautet dabei die Ausgangsthese. Die analysierten kulturwissenschaftlichen Theorien des Imaginären weisen Kohns zufolge eine durchgehend konservative politische Ideologie auf, was sich nicht zuletzt in der Behauptung einer apriorischen Notwendigkeit des Imaginären für die soziale Ordnung zeigt. Gegen die Vorstellung einer ästhetischen »Verkörperung « des politischen Imaginären in einem konkreten Medium führt eine abschließende Lektüre einer Fotografie des Wahlkämpfers Barack Obama die radikale »Möglichkeitsvielfalt« des politischen Imaginären vor Augen.

Martin Doll entwickelt in seinem Beitrag eine Theorie und Genealogie des Techno-Imaginären. In einem ersten Schritt fragt er nach dem Zusammenhang zwischen technischen Medien und dem politischen Imaginären und erarbeitet mittels einer produktiven Lektüre der Thesen Benedict Andersons in Verbindung mit Überlegungen von James Carey ein ritualistisches Modell des Mediengebrauchs. Vor diesem Hintergrund untersucht er in einem zweiten Schritt vor allem neuere, mit Social Media verbundene Vorstellungsräume politischer Gemeinwesen. In seiner Betrachtung insbesondere der Diskurse über Facebook zeigt Doll, dass sich das damit verknüpfte Techno-Imaginäre zwischen holistischen Unifizierungsphantasien einer Weltgesellschaft und einem radikal individualistischen Liberalismus bewegt.

Der Beitrag von Leander Scholz bringt Castoriadis' Theorie des politischen Imaginären in einen diskursgeschichtlichen Zusammenhang mit der Philosophie Kants. Scholz macht geltend, dass Castoriadis die historische Dimension der radikalen 
Einbildungskraft unterschlägt: Die radikal neue Schöpfung politischer Ordnung ex nihilo wird erst durch den Abbau tradierter symbolischer Ordnungen in der Aufklärung denkbar. Kants Philosophie erscheint aus dieser Perspektive genuin modern, insofern sie um die Idee einer freien (Selbst-)Gesetzgebung kreist. Scholz liest schließlich die Kritik der Urteilskraft als einen Grund text der politischen Philosophie der Moderne, in der das Schöne als Modus der Projektion einer politischen Ordnung und das Erhabene als »Immunisierung " gegen die nackte Gewalt des Realen fungieren.

Harun Maye verbindet die Politik und das Imaginäre geradezu andersherum als sonst üblich: Er analysiert - im Gegensatz zur imaginierten Politik - die politische Metaphorik in den vermögenstheoretischen Diskursen über die Einbildungskraft »um 1800 « und arbeitet heraus, dass dort bereits eine komplexe politische Philosophie mitdiskutiert wurde. In einem zweiten Schritt unterzieht Maye die Konzeption des Imaginären bei Wolfgang Iser und Karl Ludwig Pfeiffer einer kritischen Analyse und führt vor, dass beide die mediale Dimension des Imaginären ausblenden. Gegen die »Medienvergessenheit« der Theorie des Imaginären betont Maye die Angewiesenheit aller imaginären Prozesse auf mediale Techniken. Zugleich gibt er einen eher skeptischen Ausblick auf die Verwendbarkeit der Kategorie des Imaginären.

Martin Roussel fragt anhand der Lektüre von Texten Paul Ricœurs und Jacques Derridas nach der Beziehung des Imaginären zur Sprache. Im ersten Teil seiner Argumentation bezieht sich Roussel auf Ricœurs Entgegensetzung von Ideologie und Utopie. Beide konstituieren für Ricœur sprachliche Möglichkeiten des sozialen Imaginären, insofern sie eine politische Ordnung durch die Errichtung eines Gründungsereignisses legitimieren (Ideologie) bzw. der politischen Wirklichkeit eine grundsätzliche Alternative entgegenhalten (Utopie). Roussel kontrastiert diese Matrix mit einer Lektüre von Derridas Text Les yeux de la langue, in dem die Auseinandersetzung zwischen Scholem und Rosenzweig über jüdische Identität und ihre sprachliche Verfassung kommentiert wird.

Rolf Parr legt dar, wie sich politisch-nationale Ganzheiten, Kollektive und Identitäten unter Rückgriff auf die Interdiskurstheorie konzipieren lassen. Insgesamt versucht Parr damit eine methodische Antwort auf die Frage nach dem Zusammenhang zwischen einzelnen ästhetisch-politisch relevanten diskursiven Elementen des Politischen und dem politischen Imaginären zu geben. Die Interdiskurstheorie ermöglicht laut Parr nicht nur Texte 
und Dokumente ganz unterschiedlicher Herkunft, wie z. B. kunstliterarische Texte, Alltagsrede, politische Verlautbarungen sowie historisches und aktuelles Bild- und Filmmaterial, aufeinander zu beziehen. Sie erlaubt es auch, daraus zugleich mehrere kollektive und individuelle Identitäten herauszuarbeiten sowie die Friktionen zwischen ihnen zu erklären.

Der Beitrag Jan Lohls über »Die Nation als imaginäre Gemeinschaft « unternimmt eine psychoanalytisch ausgerichtete, sozialpsychologische Analyse des deutschen Nationalismus nach der deutschen Einheit. Methodisch ergänzt Lohl Benedict Andersons Konzept der »imagined communities « um Elemente der Psychoanalyse Jacques Lacans, der Symboltheorie Alfred Lorenzers sowie der Gruppenidentitätsforschung Vamik Volkans. Aus dieser Perspektive untersucht Lohl die Diskurse der »Normalisierung « in Deutschland nach der Wiedervereinigung und diskutiert diese im Rahmen der nicht aufgearbeiteten deutschen Schuld durch die NSVerbrechen. Lohl insistiert darauf, dass die Rede von einer endlich »normalisierten « deutschen Nation den weiterhin grassierenden Antisemitismus und Rassismus ausblenden muss.

Romain Jobez widmet sich in seinem Beitrag einem im deutschsprachigen Raum wenig rezipierten Werk Claude Leforts: Le travail de l'ouvre Machiavel. Jobez legt den Fokus dabei auf eine Lektüre Leforts von Gramscis Gefängnisheften, in denen Gramsci wiederum Machiavelli interpretiert. Er zeigt, dass Lefort die Gefängnishefte zum Anlass nimmt, mit und gegen Gramsci schon in $\mathrm{Il}$ Principe die Idee angelegt zu sehen, dass das Wesen der Gesellschaft nur durch »eine Quasi-Repräsentation seiner selbst« erfassbar sei: Während die Figur des Fürsten dem Volk eine imaginäre Verkörperung seiner selbst vermittle und den Ort der Macht vollständig besetze, bleibe dieser in demokratischen Gesellschaften leer und unbesetzbar, kann immer nur vorläufig und niemals restlos repräsentiert werden.

Benjamin Wihstutz plädiert in seinem Artikel dafür, Jacques Rancières Bühnenmetaphern in seinem politischen Denken ernst zu nehmen. Wenn Politik, wie Rancière schreibt, zuallererst »Konflikt über das Dasein einer gemeinsamen Bühne« ist, lässt sie sich im Kern als theatral auffassen, weil sie an eine Inszenierung gebunden wird: Denn es handelt sich um ein konflikthaftes In-SzeneSetzen eines >Anteils der Anteillosen<, um ein Erscheinenlassen von Gleichheit. Rancières Theatermetaphorik umfasst dabei beide >Seiten > des Konflikts: Aus der Perspektive der politischen Subjekte 
geht es um die Sichtbarmachung des >Unrechts $<$ ungleicher Verteilung; aus dem Blickwinkel der bestehenden sozialen Ordnung wird unbefugt eine öffentliche Bühne eingerichtet, weil so getan wird, als $o b$ es einen gemeinsamen Raum öffentlicher Teilhabe bereits gäbe.

Jan Völker beschließt den Band mit einer über Jacques Rancière und Alain Badiou vermittelten grundlegenden Kritik an einem konsensuellen Verständnis von Demokratie als Staatsform. Im Gegenzug entwirft er eine andere Seite der Demokratie, und zwar im Denken einer Differenz von Politik und Staat: Während für Völker das mit der demokratischen Staatsform verbundene liberale Imaginäre um den Glauben an den Markt mit seinen allgemeinen Äquivalenzprinzipien zirkuliert, stellt eine nicht-etatistische Politik die Universalität des Grundsatzes der Gleichheit »aller als absolut unvergleichlicher « (Nancy) auf. Schließlich fragt Völker, wie mit Badiou und gegen Althusser diese Politik ohne Staat mit einem nicht-staatlichen Imaginären zusammenzudenken wäre.

Die hier versammelten Beiträge gehen auf einen Workshop zurück, der im Januar 2011 im Rahmen des ATTRACT-Projekts »Ästhetische Figurationen des Politischen« an der Universität Luxemburg stattfand. Die Publikation bildet außerdem den Auftakt der von Martin Doll und Oliver Kohns herausgegebenen Publikationsreihe »Texte zur politischen Ästhetik«. Unser Dank geht an den >Fonds National de la Recherche (FNR) für die finanzielle Ausstattung des Projekts während seiner Laufzeit von fünf Jahren. Im Zusammenhang mit dieser ersten Veröffentlichung danken wir vor allem auch Kathrin Roussel und Stefan Claudius für die Gestaltung der Buchreihe sowie für das Setzen der Beiträge. 